\title{
Design and Implementation of an Automatic System for Dielectric Characterization of Ceramic Materials
}

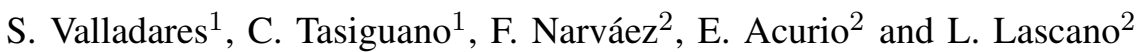 \\ ${ }^{1}$ Departamento de Automatización y Control Industrial, Escuela Politécnica Nacional, Ecuador, \\ sara.valladares@epn.edu.ec, cristian.tasiguano@epn.edu.ec \\ ${ }^{2}$ Departamento de Física, Escuela Politécnica Nacional, Ecuador, \\ fernanda.narvaez@epn.edu.ec, eliana.acurio@epn.edu.ec, luis.lascano@epn.edu.ec
}

\begin{abstract}
This article describes the design and implementation of an automatic dielectric characterization system by using the non-destructive technique of the complex impedance spectroscopy. This method allows obtaining the dielectric constant and the complex impedance for a wide range of temperatures and different frequencies of the applied field. The developed user interface eases the configuration of two impedance analyzers and the setup of the characterization test. Additionally, test reports are automatically generated from the measurements, which simplify the experimental analysis by plotting the more important parameters in the dielectric characterization. One of these parameters, the peak of the graph $\mathrm{Er}^{\prime}$ (real part of the dielectric constant) vs. temperature, which is the phase transition temperature from the ferroelectric to the paraelectric state of the ceramic materials, is obtained and compared to reference values to demonstrate the system correct functioning.
\end{abstract}

Keywords-Ceramic, complex impedance, dielectric constant, LabVIEW, PID.

\section{INTRODUCTION}

Ceramic materials are non-metallic inorganic chemical compounds widely used in many applications in almost all the engineering disciplines. In special, electroceramics are advanced materials whose properties strictly depend on the structure, processing steps and composition [1]. The desired properties can be categorized in intra-granular or "bulk" properties, intergranular or "grain boundary", a combination of both, and surface properties [2]. Therefore, a complete characterization of electroceramics requires the analysis of many aspects such as grain boundaries in polycrystalline materials, behavior differences between bulk and grain boundary region, etc.

Among the most used characterization techniques of electroceramics are microscopy, electrical measurements and complex impedance spectroscopy (CIS). Scanning electron microscopy (SEM) and transmission electron microscopy (TEM) are mainly employed to characterize the microstructure of the material under investigation, while DC electrical measurements allow establishing electric characteristics, but this is not an appropriate method to extract inter- and intra-granular resistance values [2]. On the contrary, complete information of the material topology with a clear distinction between, surface, grain boundary and bulk components and its correlation with electrical properties can be obtained with CIS technique. The impedance measurements are performed by applying an $\mathrm{AC}$ signal across the sample over a wide frequency range and the different regions of the material can be separated and characterized according to their different relaxation times or time constants [1].

Especially, this non-destructive method that elucidates the electrical nature of the ceramic material also allows performing the dielectric characterization of the material which is an important parameter to be determined in the Electroceramic Laboratory of Escuela Politécnica Nacional. Since CIS is a powerful characterization technique to analyze the electrical properties of ceramics, e.g. impedance and/or dielectric, the aim of this work is to implement this technique in an automatic system to obtain the dielectric constant of electroceramics by adapting the available measurement equipment to a more versatile and easy-to-use system.

Compared with other works [3], the developed system offers versatility to be able to perform measurements with two impedance analyzers and its management through a userfriendly interface developed in LabView.

The remainder of this paper is organized as follows: in Section II, we provide details of the theory behind CIS technique and dielectric-constant extraction; in Section III, we describe the designed automatic characterization system; in Section IV, we show and discuss the results obtained in different ceramics and finally, in Section V the main achievements of this paper are summarized.

\section{Complex Impedance Spectroscopy}

Complex Impedance Spectroscopy is a method that allows acquiring the impedance spectrum of a material. From the obtained spectrum, the dielectric constant and the conductivity of the material under analysis can be determined [3]. The general procedure starts by applying an electrical stimulus to the electrodes of the sample. This stimulus can be a voltage or a current with a known frequency and magnitude. Then, the response of the sample to this stimulus is analyzed. The technique used is a standard technique, which consists of directly measuring the impedance of the material in the frequency domain. Therefore, a fixed-frequency voltage $V=V_{0} \cdot \sin (w t)$ ( $w$ represents the angular frequency) is applied to the sample and subsequently, the phase shift and amplitude of the resultant current $i=I_{0} \cdot \sin (w t+\phi)$ can

Digital Object Identifier (DOI):

http://dx.doi.org/10.18687/LACCEI2019.1.1.29

ISBN: 978-0-9993443-6-1 ISSN: 2414-6390

$17^{\text {th }}$ LACCEI International Multi-Conference for Engineering, Education, and Technology: Industry, Innovation, And Infrastructure for Sustainable Cities and Communities, 24-26 July 2019, Jamaica. 


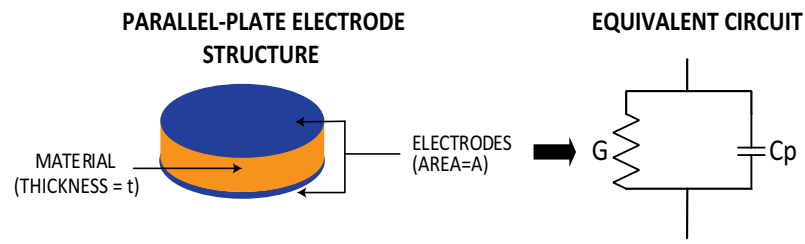

Fig. 1. Parallel plates method and its equivalent circuit.

be extracted. The magnitude and the phase are related to the resistive component and the reactive component of the impedance, respectively [2]. The magnitudes studied in CIS can be a function of frequency and temperature, but especially the relationship between the parameters and the temperature allows knowing the nature of the conduction and polarization mechanisms of the material[3], [4]. Impedance $\left(Z^{*}\right)$, therefore, is a vector defined as a complex number, whose magnitude and direction can be expressed by real $\left(Z^{\prime}\right)$ and imaginary $\left(Z^{\prime \prime}\right)$ as follows:

$$
Z^{*}=Z^{\prime}-j Z^{\prime \prime}=R-j X
$$

where $\mathrm{R}$ is the resistance and $\mathrm{X}$ the reactance. Additionally, three other complex parameters can be derived from the complex impedance:

- The complex admittance, which is defined as the inverse of the complex impedance:

$$
Y^{*}=\left(Z^{*}\right)^{-1}=Y^{\prime}+Y^{\prime \prime} .
$$

The real part of the admittance $\left(Y^{\prime}\right)$ is more frequently known as the conductivity $(\sigma)$.

- The complex electric modulus:

$$
M^{*}=j \omega C_{0} Z^{*}=M^{\prime}+M^{\prime \prime}
$$

where $C_{0}=\varepsilon_{0} A / l$ is the vacuum capacitance of the empty measuring cell with electrode area $A$, and separation 1 ; while $\varepsilon_{0}$ is the vacuum permittivity 8.854 x $10^{-14} \mathrm{Fcm}^{-1}$.

- The complex permittivity, which can be defined as the inverse of the complex electric modulus:

$$
\varepsilon^{*}=\left(M^{*}\right)^{-1}=Y^{*}\left(j \omega C_{0}\right)^{-1}=\varepsilon^{\prime}+\varepsilon^{\prime \prime} .
$$

\section{A. Dielectric constant}

As can be seen in Eq. (5), the dielectric constant $\left(K^{*}\right)$ is equivalent to the relative permittivity $\left(\varepsilon_{r}^{*}\right)$, i.e. it is equivalent to the relation between the permittivity $\left(\varepsilon^{*}\right)$ and the permittivity of the vacuum $\left(\varepsilon_{0}\right)$ [5].

$$
K^{*}=\varepsilon_{r}^{*}=\varepsilon_{r}^{\prime}-j \varepsilon_{r}^{\prime \prime}=\frac{\varepsilon^{*}}{\varepsilon_{0}}
$$

Relative permittivity describes the interaction between a material and the electric field applied to it. The real part $\left(\varepsilon_{r}^{\prime}\right)$ is related to the stored energy of an external electric field in a material and the imaginary part $\left(\varepsilon_{r}^{\prime \prime}\right)$, also called the loss

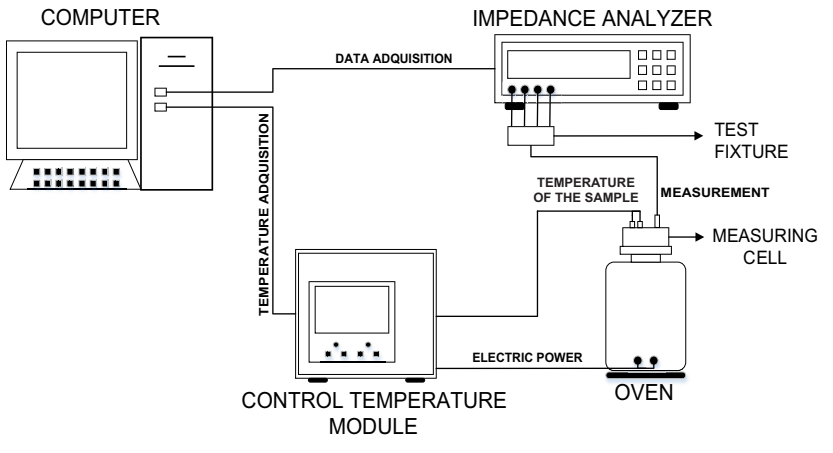

Fig. 2. Diagram of the automatic dielectric characterization system.

factor, is related to how much energy from an external field is lost or dissipated [5].

$$
K^{*}=\varepsilon_{r}^{*}=\frac{\varepsilon^{*}}{\varepsilon_{0}}
$$

\section{B. Measuring methodology of parallel plates}

The measuring method of parallel plates (or flat-parallel electrodes) is used when a measuring instrument of impedance is available, such as the HP 4263B LCR meter and the AGILENT 4294A precision analyzer used in this work. This method consists of inserting between two electrode (plates) a thin sheet of the electroceramic material under test to form a capacitor [5], as depicted in Fig. 1.

The capacitance $\left(C_{p}\right)$ and conductance $(G)$ parameters obtained by the measuring instrument are then employed to calculate the dielectric constant by using the following expressions:

$$
\begin{gathered}
\varepsilon_{r}^{\prime}=\frac{C_{p} \cdot t}{\varepsilon_{0} \cdot A} \\
\varepsilon_{r}^{\prime \prime}=\frac{G \cdot t}{\omega \cdot \varepsilon_{0} \cdot A} .
\end{gathered}
$$

\section{Automatic Dielectric Characterization SYSTEM}

The designed automatic characterization system allows determining the value of the dielectric constant and the complex impedance by using different temperatures and frequencies of the applied field. During the characterization, the sample is heated slowly in an oven at a constant heating rate and the measurements are subsequently performed every certain temperature interval while at the same time an electric field is applied at different frequencies. Fig. 2 shows the scheme of the implemented characterization system.

\section{A. Heating subsystem}

The heating subsystem consists of a temperature controller (Eurotherm 2404), an actuator (solid state power controller WATLOW DIN-A-MITE DB10-24F0-S200), a plant (electric oven) and a temperature sensor (thermocouple type K). In Fig. 3, the implemented temperature control system is illustrated as a block diagram. 


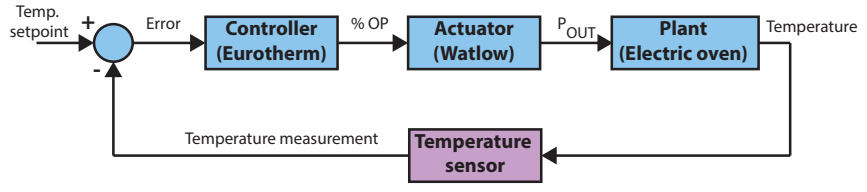

Fig. 3. Block diagram of the temperature control system. \%OP is the output power percentage and $P_{\text {out }}$ is the output power.

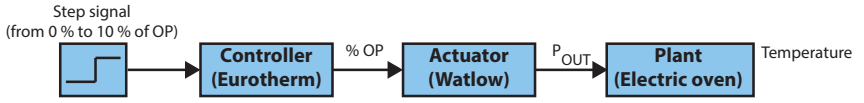

Fig. 4. Block diagram of the open loop system to extract the reaction curve.

The temperature controller is set to work with a reverse action control, i.e. an increase in the process variable PV (temperature) originates a decrease in the controller output [6]. Additionally, within the requirements of the process itself, the heating system must be capable of gradually varying the temperature in a range from room temperature to a maximum of $950{ }^{\circ} \mathrm{C}$ depending on the electroceramic sample analyzed.

To properly calibrate the controller, we first obtained a simplified model of the plant by employing the reaction curve of the system; therefore, a step signal is applied to the input of the open-loop system as depicted in Fig. 4.

The reaction curve obtained for the plant is shown in Fig. 5. The curve exhibits an "S" shape, which is characteristic of a second order or higher order processes [6]. To obtain the model, we proceed to adjust its reaction curve to a first-order transfer function with dead time as follows:

$$
\begin{gathered}
K=\frac{\Delta C_{s}}{\Delta m} \\
\tau=\frac{3}{2}\left(t_{2}-t_{1}\right) \\
t_{0}=t_{2}-\tau \\
G_{1}(s)=\frac{K}{\tau s+1} e^{-t_{0} s}=\frac{38.24}{22.65 s+1} e^{-2.75 s}
\end{gathered}
$$

where $\Delta C_{s}$ is the output-signal variation of the plant, $\Delta m$ is the output-signal variation of the controller (from $0 \%$ to $10 \%$ of OP), $K$ is the steady-state gain, $t_{0}$ is the dead time and $\tau$ is the time constant of the process. The parameter $\Delta C_{s}$ is extracted directly from the reaction curve, while $t_{1}$ and $t_{2}$ are the time values at which $\Delta C_{s}$ reaches the $28.3 \%$ and $62.3 \%$, respectively.

Once we obtained the model of the plant, the structure of the controller was selected. Since the transfer function corresponds to a first order system with dead time, it is recommended to use a proportional-integral controller (PI) [7],[8]. Subsequently, the parameters of this controller are obtained by using the tuning formulas of the Ziegler-Nichols method as follow:

$$
\begin{aligned}
K_{c} & =\frac{0.9}{K}\left(\frac{t_{0}}{\tau}\right)^{-1}=0.194 \\
T_{i} & =3.33 t_{0}=9.16 \mathrm{~min}
\end{aligned}
$$

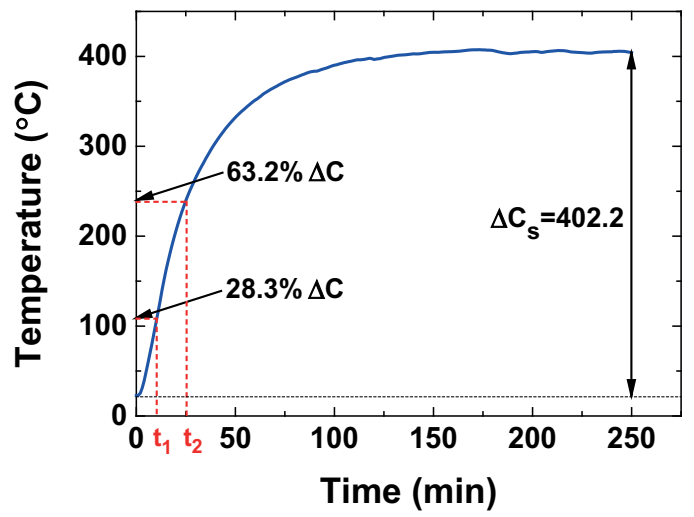

Fig. 5. Reaction curve and extraction of the model parameters $\left(\Delta C_{s}, t_{1}\right.$ and $\left.t_{2}\right)$.

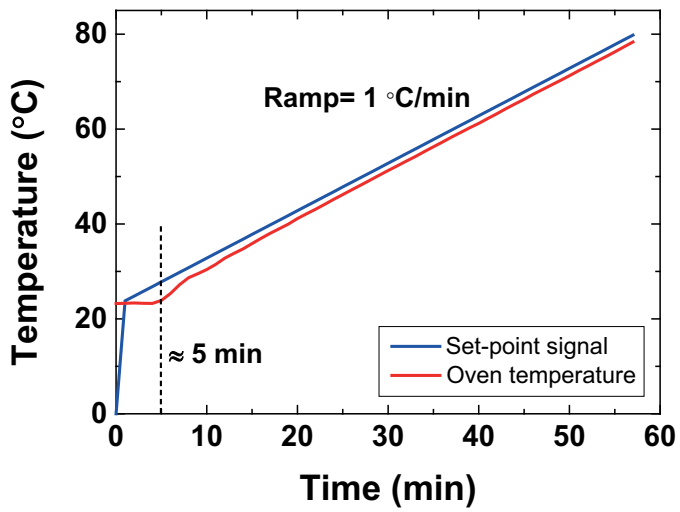

Fig. 6. Response of the system to a heating ramp.

where $K_{c}$ and $T_{i}$ are the proportional gain and the integral time, respectively. Instead of $K_{c}$, the proportional band $(P B)$ is a more common parameter used in commercial controllers [6] such as the Eurotherm controller. $P B$ expresses the gain of the controller as a percentage of the instrument range [9] and is related to $K_{c}$ through $P B \%=100 / K_{c}$. With the resulting values $\left(P B=515.46\right.$ and $\left.T_{i}=9.16 \mathrm{~min}\right)$, the controller response takes approximately 30 minutes to follow the temperature setpoint with a constant heating rate of $1{ }^{\circ} \mathrm{C} / \mathrm{min}$ (not shown). For this reason, fine tuning was performed manually based on the values previously calculated. As a result, $P B$ and $T_{i}$ were reduced to 100 and $8.6 \mathrm{~min}$, respectively, to decrease the response time of the system as depicted in Fig. 6. It can be seen that the response time with these new values is approximately five minutes, which is significantly shorter than the previous value.

The setpoint configurator of the Eurotherm temperature controller saves a total of four programs and within each one a maximum of 16 segments [10], which are used to define the reference signal. The segments used in this work are ramp, $d w e l l$, and end. In the ramp segment, the setpoint increases 


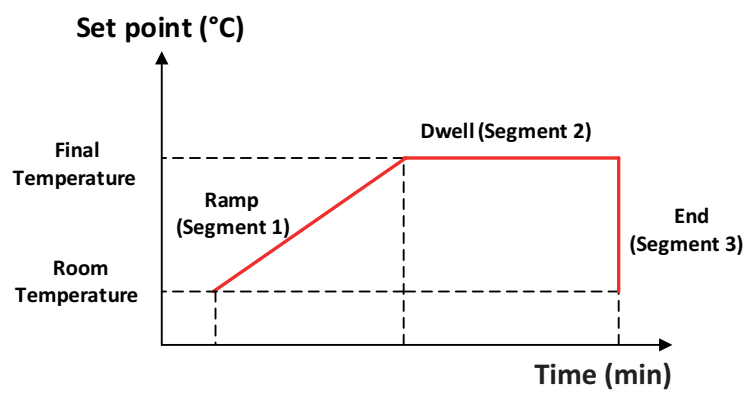

Fig. 7. Setpoint signal by using the programmable segments in the Eurotherm controller.

TABLE I

MESSAGE STRUCTURE IN THE MODBUS PROTOCOL.

\begin{tabular}{|l|l|l|l|}
\hline \hline Device address & Function code & Data & Error checking \\
\hline
\end{tabular}

TABLE II

BIT CONFIGURATION OF THE GPIB ADDRESS.

\begin{tabular}{|c|c|c|c|c|c|c|c|c|}
\hline \hline Bit position & 7 & 6 & 5 & 4 & 3 & 2 & 1 & 0 \\
\hline Meaning & 0 & TA & LA & \multicolumn{6}{|c|}{ GPIB address (0-30 range $)$} \\
\hline \hline
\end{tabular}

linearly; in the $d w e l l$ segment, the setpoint remains constant for a specific period of time, and the end segment stops the program finishing the heating process as shown in Fig. 7.

\section{B. Communication subsystem}

To automatically perform the acquisition of both the oven temperature (Eurotherm controller) and the measurements that allow the dielectric calculation (impedance analyzer), it is necessary to implement a communication network between all the equipment that constitutes the system (see Fig. 2).

The computer is the main component of this network because is the master of the Modbus protocol for the communication with the Eurotherm controller and controls the General Purpose Interface Bus (GPIB) for the communication with the impedance analyzers (the HP 4263B LCR meter or the AGILENT 4294A precision analyzer).

- Modbus protocol: It is a serial communication protocol that defines a digital communication network that has one master and one or more slaves. A typical transaction consists of sending a request from the master followed by a response from the slave [11]. A message sent between the master and the slave in any direction requires the information detailed in Table I. It is worth noting that each slave has a unique address of 8 bits; however, the address ' 0 ' is a special case in which a message is sent to all the slaves of the network.

The Eurotherm temperature controller allows the user to configure data transmission with integer resolution (16-bit integer) or full resolution (32-bit floating-point number) [11]. In this work, the full resolution is used to obtain the data of the temperature measurements in decimal format.

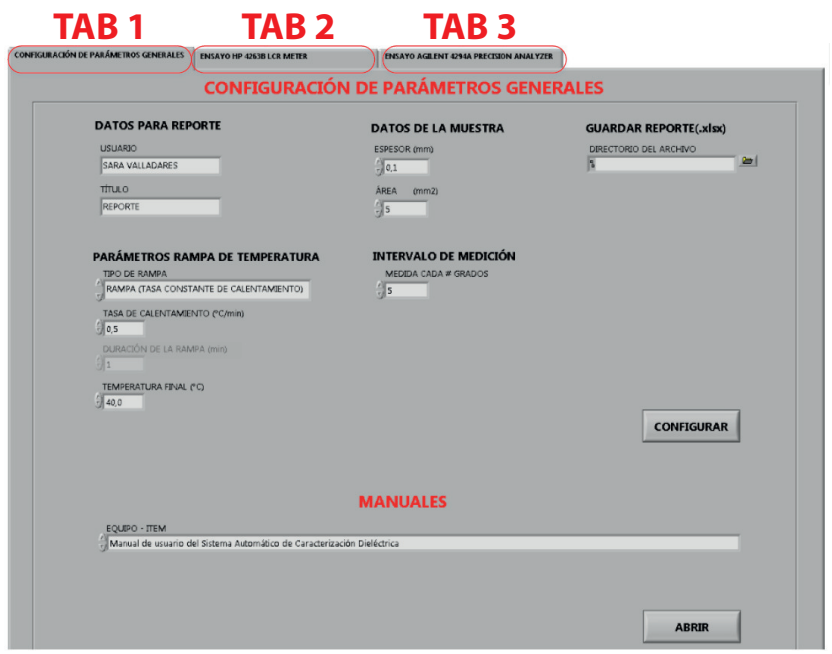

Fig. 8. User Interface.

- GPIB: The ANSI / IEEE 488.1-2003 standard, also known as GPIB, is a digital 8-bit parallel communication interface that describes a standard communication interface for instruments and controllers from different manufacturers [12]. The GPIB devices can be talkers, listeners or controllers. The bus controller manages the data flow, defines the communication links and sends the GPIB commands to the instruments. Several controllers may exist on the bus, but only one active controller is allowed at a time. The GPIB supports a system controller and a total of 14 additional instruments[12].

All GPIB devices must be assigned with a single GPIB address, which is in a range from 0 to 30 . The bus controller uses this address to designate which devices should be listening or talking at any given time. The talker address is defined by setting the TA (Talk Active) bit, while the listener address is defined by setting the LA (Listen Active) bit of the GPIB address. The bit configuration of the GPIB address is illustrated in Table II [12].

\section{User Interface}

The user interface was implemented with the software LabVIEW, which is a system-design platform and development environment from National Instruments. It uses a visual/graphical programming language that eases the handling of communication interfaces such as serial port, GPIB, etc. Moreover, LabVIEW has a great variety of libraries that reduces the time of software development for engineering. The programs developed in LabVIEW are normally called VIs (Virtual Instruments).

It is worth mentioning that the implementation of the communication within the user interface has been developed by using the LabVIEW Instrument Drivers. They are sets of highlevel functions that simplify the communication and control of a programmable instrument. Each one of these functions allows the initialization, configuration and data acquisition 

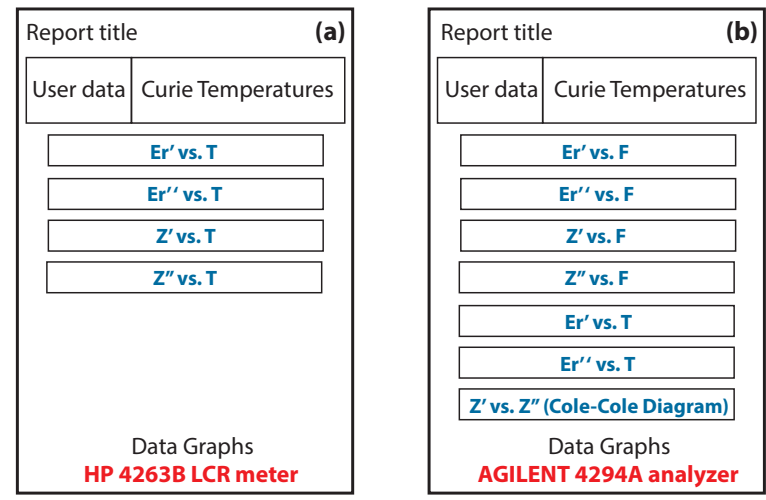

Fig. 9. Data Graph sheet for (a) the HP 4263B analyzer and (b) AGILENT 4294A precision analyzer.

from the instruments. We have in total six categories to control specifics functions of the instrument.

1) Initialize VI: This VI establishes communication with the instrument and must be the first called VI.

2) Configure VIs: They are a set of software routines that configure the instrument to perform specific operations.

3) Action/Status VIs: These VIs start or end measurement operations since they order the instrument to perform an action based on its current configuration. Status VIs obtain the current status of the instrument or the status of pending operations.

4) Data VIs: They transfer data to or from the instrument.

5) Utility VIs: They perform a variety of auxiliary operations (reset, self-test), and may include other custom routines, such as calibration or storage and recovery of instrument configurations.

6) Close VI: This VI ends the communication with the instrument.

Moreover, the user interface has a presentation screen and a window which contains three tabs to perform the characterization. The first tab allows the configuration of general parameters (user data for report header, ramp type, heating rate, final temperature, etc.) and gives the access to the equipment manuals. The second and third tab allow testing with the HP 4263B LCR meter and the AGILENT 4294A precision analyzer, respectively. Fig. 8 shows these three tabs.

The main measures that are acquired from the impedance analyzers during the tests are equivalent parallel capacitance $\left(C_{p}\right)$ and quality factor $(D)$; conductance $(G)$ and susceptance $(B)$; and impedance $(Z)$. The value of the dielectric constant (real part and imaginary part) is then obtained from these measurements by using equations (6) and (7).

\section{Automatic reports}

To obtain adequate handling of the data obtained in the different tests, the reports are generated in Microsoft Excel by using the Report Generation Toolkit of LabVIEW. Each test report contains two sheets. The first one is called Data Table and contains the measurements of all the aforementioned parameters. The second one is called Data Graphs and depicts

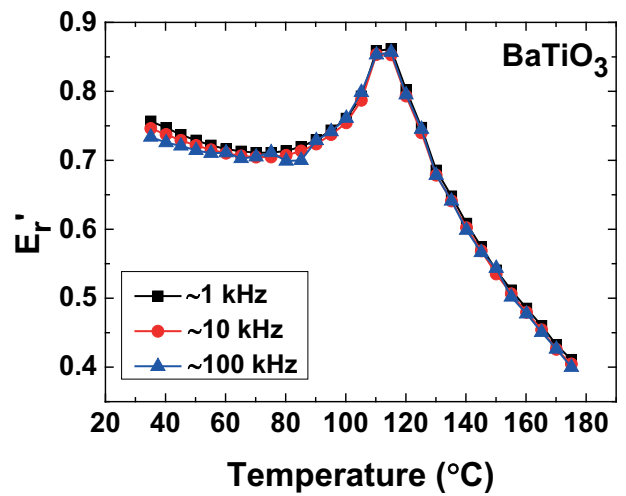

Fig. 10. $E r^{\prime}$ as a function of the temperature of the $\mathrm{BaTiO}_{3}$ sample obtained with the AGILENT 4294A precision analyzer.

TABLE III

Phase transition TEMPERATURE IN A SAMPLE OF $\mathrm{BaTiO}_{3}$ AT DIFFERENT FREQUENCIES BY USING THE AGILENT 4294A PRECISION ANALYZER.

\begin{tabular}{|c|c|c|c|}
\hline \multicolumn{4}{|c|}{$\mathrm{BaTiO}_{3}$} \\
\hline & $974.12 \mathrm{~Hz}$ & $9965.1 \mathrm{~Hz}$ & $100 \mathrm{kHz}$ \\
\hline Tc $\left({ }^{\circ} \mathrm{C}\right)$ & 115.09 & 115.09 & 115.09 \\
\hline $\mathbf{E r}_{\max }^{\prime}$ & 0.872 & 0.864 & 0.857 \\
\hline Absolute error & 9.91 & 9.91 & 9.91 \\
\hline Error \% & 7.928 & 7.928 & 7.928 \\
\hline
\end{tabular}

the real and the imaginary part of the dielectric constant $\left(E r^{\prime}, E r^{\prime \prime}\right)$ and the impedance $\left(Z^{\prime}, Z^{\prime \prime}\right)$ as a function of the temperature for the five fixed frequencies that are available in the HP 4263B analyzer.

Since there are not fixed frequencies in the AGILENT $4294 \mathrm{~A}$ precision analyzer but the user configures the range of frequencies instead, it is also possible to graph the real and the imaginary part of the dielectric constant $\left(E r^{\prime}, E r^{\prime \prime}\right)$ and the impedance $\left(Z^{\prime}, Z^{\prime \prime}\right)$ as a function of the frequency. It is important to notice that for each graph, three temperatures were used (the initial, the average and the final temperature) since they are enough to visualize the sample behavior. Additionally, the real part of the impedance based on the imaginary part of the impedance (with a negative sign) for the entire frequency range (Cole-Cole diagram) is also plot in this sheet. Fig. 9 illustrates an schematic of the Data Graphs sheet for each impedance analyzer.

\section{RESULTS AND DisCUSSION}

Since the parameters to perform the dielectric characterization $\left(Z, C_{p}\right.$ and $\left.G\right)$ depend on several factors such as the purity and porosity of the sample, the quality of the electrodes, the sample dimensions, etc., we verified the data obtained from the reports generated by the automatic dielectric characterization system by means of the phase transition temperature from the ferroelectric to the paraelectric state. This temperature is also known as Curie temperature and is extracted from the 


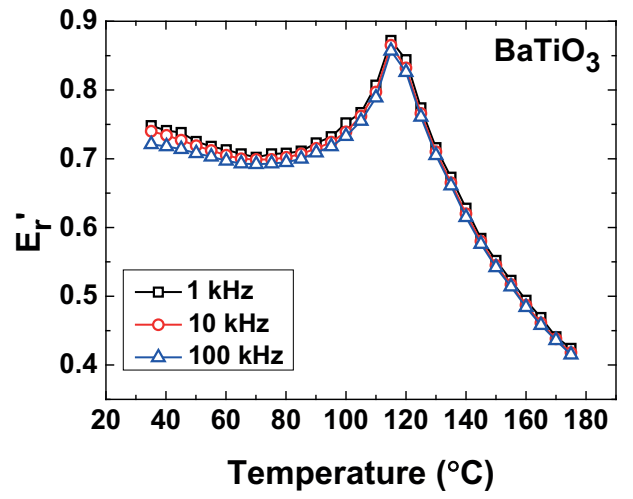

Fig. 11. $E r^{\prime}$ as a function of the temperature of the $\mathrm{BaTiO}_{3}$ sample obtained with the HP 4263B LCR meter analyzer.

TABLE IV

Phase Transition TEMPERATURE IN A SAMPLE OF $\mathrm{BaTiO}_{3}$ AT DIFFERENT FREQUENCIES BY USING THE HP 4263B LCR METER ANALYZER.

\begin{tabular}{|c|c|c|c|}
\hline \hline \multicolumn{4}{|c|}{$\mathrm{BaTiO}_{\mathbf{3}}$} \\
\hline & $\mathbf{1 ~} \mathbf{~ k H z}$ & $\mathbf{1 0} \mathbf{~ k H z}$ & $\mathbf{1 0 0} \mathbf{~ k H z}$ \\
\hline $\left.\mathbf{T c ~}^{\circ} \mathbf{C}\right)$ & 115.10 & 110.20 & 115.10 \\
\hline $\mathbf{E r}_{\text {max }}^{\prime}$ & 0.861 & 0.853 & 0.856 \\
\hline Absolute error & 9.9 & 14.8 & 9.9 \\
\hline Error \% & 7.92 & 11.84 & 7.92 \\
\hline \hline
\end{tabular}

maximum value of the dielectric constant, i.e. the peak of the curve $E r^{\prime}$ vs Temperature [3]. We use this value because it is not related to the size of the sample, but to its composition. Therefore, samples of the same compound will have similar phase transition temperatures.

To compare the results, we used as reference values the phase transition temperatures obtained from the Literature for the same compounds provided by the laboratory [13]. One of the samples analyzed was a barium titanate $\left(\mathrm{BaTiO}_{3}\right)$ compound with a thickness of $0.6 \mathrm{~mm}$ and an area of 38.48 $\mathrm{mm}^{2}$. The curve $E r^{\prime}$ vs temperature for the $\mathrm{BaTiO}_{3}$ sample was obtained by heating the oven from room temperature to $180{ }^{\circ} \mathrm{C}$ to visualize the peak value of the dielectric constant (real part) and obtain the Curie temperature. The test was performed with the AGILENT 4294A precision analyzer for frequencies close to $1 \mathrm{KHz}, 10 \mathrm{KHz}$ and $100 \mathrm{KHz}$ and the curves $E r^{\prime}$ vs Temperature are shown in Fig. 10.

According to [13] the phase transition temperature from the ferroelectric to the paraelectric state of the $\mathrm{BaTiO}_{3}$ compound is a value close to $125^{\circ} \mathrm{C}$. Table III lists the Curie temperatures obtained in this test and the absolute error calculated using 125 ${ }^{\circ} \mathrm{C}$ as the reference. Similar results were also obtained with the HP 4263B LCR meter analyzer for the fixed frequencies of $1 \mathrm{KHz}, 10 \mathrm{KHz}$ and $100 \mathrm{KHz}$ as shown in Fig. 11 and Table IV.

As can be seen in Table III and Table IV, the phase transition temperature from the ferroelectric to the paraelectric state obtained for the $\mathrm{BaTiO}_{3}$ sample with both analyzers is similar and has a value very close to that one specified in the Literature.

\section{CONCLUSions}

In this work, we have designed and implemented an automatic dielectric characterization system, which allows controlling the temperature throughout the measuring range, establishing the communication between two different impedance analyzers and the computer, performing the data acquisition of the impedance and the dielectric constant, and finally, generating reports based on the data stored during the development of the characterization test. The values of the phase transition temperature obtained for the $\mathrm{BaTiO}_{3}$ compound are in good agreement with the values extracted from the Literature, which demonstrates the correct functioning of the developed system.

\section{REFERENCES}

[1] J. Irvine, D. Sinclair and A. West, "Electroceramics: Characterization by Impedance Spectroscopy," ChemInform, vol. 21, no. 26, 1990. DOI: 10.1002/chin. 199026349

[2] D. C. Sinclair, "Characterization of Electro-materials using ac Impedance Spectroscopy, Boletn la Soc. española Cermica y Vidr., vol. 65, pp. 5566, 1995.

[3] P. Durán, "Propiedades Ferro Eléctricas de Materiales Cerámicos con Estructura Tipo Aurivillius de Composiciones Basadas en $\mathrm{Bi}_{2} \mathrm{Sr} \mathrm{Nb}_{2}$ O9," Univesidad Autónoma de Madrid, 1997.

[4] E. Barsoukov and J. R. Macdonald, Impedance spectroscopy theory, experiment, and applications. Hoboken, NJ: Wiley-Interscience, 2005.

[5] Keysight Technologies, "Solutions for Measuring Permittivity and Permeability with LCR Meters and Impedance Analyzers." [Online]. Available: http://literature.cdn.keysight.com/litweb/pdf/5980-2862EN.pdf. [Accessed Jan. 15, 2019].

[6] C. A. Smith and A. B. Corripio, Principles and practice of automatic process control. Hoboken, NJ: Wiley, 2006.

[7] K. J. Aström and R. M. Murray, Feedback systems: an introduction for scientists and engineers. Princeton: Princeton University Press, 2011.

[8] S. Skogestad, "Simple analytic rules for model reduction and PID controller tuning," Journal of Process Control, vol. 13, no. 4, pp. 291309, 2003.

[9] Eurotherm, "PID Control and Controller Tuning Techniques," [Online]. Available: http://www.dcnz.com/resources/tutorials/pid.pdf. [Accessed Jan. 15, 2019]

[10] Eurotherm, "Models 2408 and 2404 Process Controller User Manual," Aug. 2014 [Revised Jan. 15, 2019]

[11] Eurotherm, "Series 2000 Communication Handbook," [Revised Jan. 15, 2019].

[12] National Instruments, "GPIB Messages", [Online]. Available: http://www.ni.com/white-paper/3389/en/. [Accessed Jan. 15, 2019]

[13] V. Petrovsky, T. Petrovsky, S. Kamlapurkar, and F. Dogan, "Dielectric Constant of Barium Titanate Powders Near Curie Temperature," Journal of the American Ceramic Society, vol. 91, no. 11, pp. 35903592, 2008. DOI: 10.1111/j.1551-2916.2008.02693.x. 